\title{
Low Plasma Hdl Cholesterol and Elevated C Reactive Protein further Increase Cardiovascular Disease Risk in Latinos with Type 2 Diabetes
}

\author{
Mariana C Calle ${ }^{1,2}$, Sonia Vega-López ${ }^{2,4}$, Sofia Segura-Pérez ${ }^{2,5}$, Jeff S Volek ${ }^{1,2,3}$, Rafael Pérez-Escamilla ${ }^{1,2,6}$ and Maria Luz Fernandez ${ }^{1,2,3 *}$
}

${ }^{1}$ Department of Nutritional Sciences, University of Connecticut, 3624 Horsebarn Rd Ext, CT 06269, USA

${ }^{2}$ Connecticut NIH EXPORT Center for Eliminating Health Disparities among Latinos, CT, USA

${ }^{3}$ Department of Kinesiology, University of Connecticut, Storrs, CT, USA

${ }^{4}$ Healthy Lifestyles Research Center at Arizona State University, Mesa, AZ, USA

${ }^{5}$ Hispanic Health Council, Hartford, CT, USA

${ }^{6}$ Yale School of Public Health, New Haven Connecticut, USA

\begin{abstract}
The purpose of this study was to determine whether low plasma HDL and high $\mathrm{C}$ reactive protein (CRP) concentrations would further increase cardiovascular disease (CVD) risk in Latinos with poorly controlled type-2 diabetes, already at high risk for CVD. Subjects $(n=68)$ were grouped into High-HDL $(\geq$ or 1.03 or $1.3 \mathrm{mmol} / \mathrm{L})$ or Low-HDL $(<1.03$ or $1.3 \mathrm{mmol} / \mathrm{L}$ ) for men and women, respectively. Following classification, risk factors for CVD including apolipoproteins, lipoprotein size and subfraction distribution were assesed. Similarly, participants were divided according to their CRP levels $(\geq$ or $<3 \mathrm{mg} / \mathrm{L}$ ) and key inflammatory markers as well as leptin and adiponectin were analyzed. The Low-HDL group had higher concentrations of the atherogenic particles, large and medium VLDL and the smaller LDL subfractions compared to the High-HDL group $(p<0.001)$. Consistently, VLDL diameter was larger and LDL diameter smaller in the Low HDL group $(p<0.001)$. The High-CRP group had larger waist circumference $(p<0.001)$ and body mass index $(p<0.001)$ than the Low-CRP group. Leptin was also higher in the High-CRP group $(p<0.01)$. These data suggest that Latinos with type-2 diabetes having either Low-HDL or High-CRP concentrations are at a higher risk for atherosclerosis and CVD than their counterparts who have High-HDL or Low-CRP.
\end{abstract}

Keywords: Type 2 diabetes; Latinos; Heart disease risk; HDL; CRP; Inflammatory markers; Apolipoproteins

Abbreviations: Apo: apolipoprotein; CVD: cardiovascular disease; HDL-C: HDL cholesterol; IL-6: interleukin 6; sICAM-1: soluble intercellular adhesion molecule-1 MCP-1: monocyte; PA: physical activity; chemotactic protein-1; T2D: type 2 diabetes; TNF- $\alpha$ : tumor necrosis factor alpha; WC: waist circumference

\section{Introduction}

Cardiovascular diseases (CVD) remain the leading cause of death in the US despite the efforts in prevention and treatment [1]. Low plasma HDL cholesterol (HDL-C) is consistently associated with increased risk of atherosclerotic disease [2]. In addition to the well defined role in reverse cholesterol transport, HDL has other protective functions. In healthy individuals HDL has anti-thrombotic[3], antioxidant [4] anti-inflammatory [5] and nitric oxide dependent vascular relaxation effects [6]. Individuals diagnosed with diabetes have low levels or impaired HDL metabolism [7]. Thus, the lack of these protective effects is one of the factors increasing their CVD risk.

Assessment of apolipoproteins, lipoprotein particle size and number allows a further characterization of CVD risk [8]. The phenotype Pattern B is described as a more atherogenic lipoprotein profile due to the higher proportion of plasma large VLDL which leads to the presence of smaller and denser LDL particles [9]. Small LDL particles are more prone to oxidation and to uptake by the arterial wall contributing to the progression of atherosclerosis [10]. Despite having plasma lipids within the normal range, people with atherogenic lipoprotein profile can be at increased CVD risk.

Atherosclerosis is characterized by chronic inflammation affecting the arterial intima [11]. Therefore certain cytokines and chemokines can be considered markers of CVD risk as well [12]. For instance, $C$ reactive protein (CRP) is a marker of the inflammatory state associated with diabetes and CVD prediction [13]. CRP is synthesized mostly by the liver upon stimulation by tumor necrosis factor alpha (TNF- $\alpha$ ) and interleukin 6 (IL-6) [14]. CRP increases the endothelial cells production of adhesion and chemoattractant molecules, such as soluble intercellular adhesion molecule-1 (ICAM-1) [15] and monocyte chemotactic protein-1 (MCP-1) respectively [16]. These molecules are involved in the development of atherosclerosis [17]. In contrast, the adipokine adiponectin is associated with insulin sensitivity and reduced risk for atherosclerosis [18].

The prevalence of Type 2 diabetes (T2D) in Hispanics compared to non Hispanic whites was $11.6 \%$ and $6.9 \%$ respectively according to the National Health and Nutrition Examination Survey (NHANES) 2003-2004 data [19]. T2D is linked with a 2 to 4 -fold higher risk of CVD, as well as an increased risk of mortality by up to 3-fold [20]. Taking into account the high proportion of Latinos with diabetes and the higher CVD risk inherent to this disease, further characterization of this population is warranted. T2D is associated with dyslipidemia [21] (specifically high triglycerides and low HDL-C levels), with the risk of endothelial dysfunction and with chronic inflammation

*Corresponding author: Maria Luz Fernandez, Department of Nutritional Sciences, University of Connecticut 3624 Horsebarn Rd Ext, Storrs, CT 06269 USA, Tel: (860)486-5547; Fax (860) 486-3674; E-mail: maria-luz.fernandez@ uconn.edu

Received October 14, 2010; Accepted November 08, 2010; Published November 10,2010

Citation: Calle MC, Vega-López S, Segura-Pérez S, Volek JS, Pérez-Escamilla R, et al. (2010) Low Plasma Hdl Cholesterol and Elevated C Reactive Protein further Increase Cardiovascular Disease Risk in Latinos with Type 2 Diabetes. J Diabetes Metab 1:109. doi:10.4172/2155-6156.1000109

Copyright: ( 92010 Calle MC, et al. This is an open-access article distributed under the terms of the Creative Commons Attribution License, which permits unrestricted use, distribution, and reproduction in any medium, provided the original author and source are credited. 
Citation: Calle MC, Vega-López S, Segura-Pérez S, Volek JS, Pérez-Escamilla R, et al. (2010) Low Plasma Hdl Cholesterol and Elevated C Reactive Protein further Increase Cardiovascular Disease Risk in Latinos with Type 2 Diabetes. J Diabetes Metab 1:109. doi:10.4172/21556156.1000109

Page 2 of 9

[22]. All these factors play a role in the increased atherosclerosis development in T2D patients. Characterizing the risk factors for CVD in Latinos diagnosed with T2D can assist with the development of more appropriate interventions.

We hypothesized that those Latinos with higher HDL-C or lower CRP would have lower risk for CVD and atherosclerosis. Thus the aims of this study were two-fold: 1) to determine whether plasma low HDL-C levels would be associated with a more atherogenic lipoprotein particle profile and therefore increased risk for atherosclerosis in Latinos with T2D and 2) to evaluate whether a higher inflammatory status, specifically high CRP levels, would further increase the risk factors for CVD in this Latino population.

\section{Materials and Methods}

This study was approved by the Institutional Review Boards of the University of Connecticut, Hartford Hospital, and the Hispanic Health Council. Baseline characteristics of anthropometrics, plasma lipids, diet and apolipoproteins were analyzed in 130 Latinos (96 women and 34 men) diagnosed with T2D at the time of enrollment in the Diabetes among Latinos Best Practices Trial (DIALBEST). This subgroup of 130 subjects was selected based on the order of enrollment and they were used for gender comparisons and traditional CVD risks factors analyses. DIALBEST, described elsewhere [23], is a parallel randomized longitudinal study with a peer counseling intervention to improve the management of T2D in this Latino population. The inclusion criteria consisted of: Latinos older than 21, diagnosed with T2D, residents of Hartford, CT, participants of "Amigos en Salud" program ("Friends in Health") at Hartford Hospital and with a glycosylated hemoglobin (HbA1c) $\geq 7 \%$. All participants signed an informed consent before enrollment. The study was conducted in the Hartford area and all survey data collection and fasting blood draws took place at the participants' household by bilingual trained personnel.

\section{Anthropometrics and blood pressure}

Waist circumference (WC) was measured to the nearest $0.1 \mathrm{~cm}$ at the midpoint between the lowest rib and the iliac crest using a flexible tape over one layer of light clothes. Body weight was measured to the closest $0.1 \mathrm{~kg}$ on a calibrated digital scale with participants wearing light clothes. Height was measured to the closest $0.1 \mathrm{~cm}$ on a portable stadiometer/scale. Body mass index (BMI) was calculated by dividing weight in $\mathrm{kg}$ by the height in $\mathrm{cm}$ squared. Blood pressure was measured on the left arm (unless contraindicated) using a manual sphygmomanometer, with the study participant in a sitting position following $5 \mathrm{~min}$ rest. All anthropometric and blood pressure measurements were collected in triplicate.

\section{Diet, Physical Activity (PA) and medications}

Bilingual trained personnel interviewed the participants to complete one 24-h dietary recall. This type of dietary assessment consists in reporting the intake of solids and liquids on the previous day. The dietary intake was analyzed using the Nutritional Data System for Research (NDSR) 5.0 (Minneapolis, MN). The diet data was entered by personnel with knowledge both in Spanish and nutrition.

Physical activity (PA) was assessed using a self reported PA frequency questionnaire. The specific question from the questionnaire used to evaluate PA in the present study was whether they perform PA for at least 30 minutes on 5 or more days per week.

Participants completed an extensive survey including open and close format questions. In our study, the hypoglycemic drugs and insulin data was obtained from two closed ended question: Are you currently taking diabetic pills? Or are you currently using insulin? The data for lipid-lowering medications was collected during the interview. Bilingual interviewers obtained medications lists by copying names and doses of drug directly from the bottles of medications in the home of participants.

\section{Plasma Glucose and Glycosylated Hemoglobin (Hba1c)}

Fasting blood was collected from the antecubital vein into EDTA and sodium fluoride-containing evacuated tubes. Plasma was separated by centrifugation at $2200 \times \mathrm{g}$ for $30 \mathrm{~min}$. at $4^{\circ} \mathrm{C}$ and immediately transported with refrigeration by authorized personnel to the University of Connecticut to be stored at $-80^{\circ} \mathrm{C}$ until analyzed. Fasting plasma glucose was measured on an automated lactate/ glucose analyzer (2300 STAT, YSI, Yellow Springs, OH). HbA1C was assessed at the participants' household from capillary blood using a disposable point-of-care Metrika A1cNow device (Metrika Inc, Sunnyvale, CA) following manufacturer's instructions. This kit is certified by the National Glycohemoglobin Standardization Program (NSGP)[24].

\section{Plasma lipids}

Aprotinin $(0.5 \mathrm{~mL} / 100 \mathrm{~mL})$, sodium azide $(0.1 \mathrm{~mL} / 100 \mathrm{~mL})$ and phenylmethylsulfonyl fluoride $(0.1 \mathrm{~mL} / 100 \mathrm{~mL})$ were added to preserve the plasma. Plasma total cholesterol, LDL-C, HDL-C and triglycerides (TG) were measured by enzymatic methods [25]. The plasma HDL-C assay is based on the analysis of the cholesterol from the supernatant after apo B containing lipoproteins precipitation. LDL-C was calculated by the Friedewald equation [26].

\section{Lipoprotein particle size and number}

Nuclear magnetic resonance (NMR) analysis was performed on a $400-\mathrm{MHz}$ NMR analyzer (Bruker BioSpin, MA, USA) as previously described [27]. Briefly, lipoprotein subclasses of different sizes produce a distinct lipid methyl signal the amplitude of which is directly proportional to lipoprotein particle concentration. NMR simultaneously quantifies $\geq 30$ lipoprotein subclasses that are empirically grouped into nine smaller subclasses based on particle diameters: large VLDL ( $\geq 60 \mathrm{~nm})$, medium VLDL (27-35 nm), small VLDL (23-27 nm), intermediate-density lipoprotein (IDL), large LDL (21.2-23 nm), medium LDL (19.8-21.2), small LDL (18-19.8 nm), large HDL $(8.8-13 \mathrm{~nm})$, medium HDL $(8.2-8.8 \mathrm{~nm})$ and small HDL $(7.3-8.2$ $\mathrm{nm})$. Weighted average lipoprotein particle sizes were calculated based on the diameter of each lipoprotein subclass multiplied by its respective relative concentration [27].

\section{Apolipoproteins}

Apolipoprotein (Apo) AI, Apo B, Apo CII, Apo CIII and Apo E were measured by duplicate using xMAP ${ }^{\circledR}$ technology on the Luminex ${ }^{\circledR}$ IS 200 system. The technique uses fluorescently labeled microsphere beads with antibodies to each individual apolipoprotein [28].

\section{IL-6, TNF-A, CRP, adiponectin, leptin, insulin, sicam-1 and MCP-1}

IL-6, TNF- $\alpha$, CRP, adiponectin, leptin, insulin, sICAM-1 and MCP1 were measured in duplicate using $\mathrm{xMAP}{ }^{\circledR}$ technology on the Luminex ${ }^{\circledR}$ IS 200 system with antibodies to the each biomarker. The sensitivities for this assay were 1.6, 50.9, 85.4 and $0.14 \mathrm{pg} / \mathrm{mL}$ for IL-6, insulin, leptin, MCP-1 and TNF- $\alpha$ respectively. Finally, for CRP, 
Citation: Calle MC, Vega-López S, Segura-Pérez S, Volek JS, Pérez-Escamilla R, et al. (2010) Low Plasma Hdl Cholesterol and Elevated C Reactive Protein further Increase Cardiovascular Disease Risk in Latinos with Type 2 Diabetes. J Diabetes Metab 1:109. doi:10.4172/21556156.1000109

Page 3 of 9

sICAM-1 and adiponectin the sensitivities were 6,9 and $56 \mathrm{pg} / \mathrm{mL}$ respectively.

\section{Statistical analyses}

Data are shown as mean \pm SD. Independent student's $t$ test was used when the data was normally distributed such as for HDL-C comparisons of dependent variables (lipoprotein particles number and size). When data was not normally distributed a non parametric test for independent samples was used. Thus, MannWhitney test was used for gender comparisons of continuous variables (anthropometrics, plasma lipids, apolipoproteins and diet) and for CRP comparisons of dependent variables (leptin, IL-6, etc). Chi square was used for comparisons of categorical variables (gender and PA or CRP high or low and gender). Data were analyzed using the Statistical Program for the Social Sciences 15.0 (SPSS) for Windows (SPSS, Inc., Evanston, IL). Results were considered significant with

\begin{tabular}{|c|c|c|}
\hline Parameter & Women $(n=96)$ & Men $(n=34)$ \\
\hline Age (years) & $55.9 \pm 11.3$ & $56.3 \pm 11.0$ \\
\hline Height (m) & $1.55 \pm 0.7$ & $1.66 \pm 0.6^{*}$ \\
\hline Weight (kg) & $82.60 \pm 21.0$ & $91.6 \pm 23.0^{*}$ \\
\hline BMI $\left(\mathrm{kg} / \mathrm{m}^{2}\right)$ & $34.3 \pm 8.1$ & $33.0 \pm 7.4$ \\
\hline WC (cm) & $106.8 \pm 16.0$ & $109.7 \pm 17.4$ \\
\hline Self-reported physically active (\%): $30 \mathrm{~min}$ of $P A \geq 5$ times $x$ week & 32.3 & $61.8^{*}$ \\
\hline Systolic $(\mathrm{mm} \mathrm{Hg})$ & $120.8 \pm 15.4$ & $120.9 \pm 15.3$ \\
\hline Diastolic (mm Hg) & $68.3 \pm 10.2$ & $78.8 \pm 9.6$ \\
\hline Glucose (mmol/L) & $10.9 \pm 4.9$ & $10.4 \pm 4.0$ \\
\hline HbA1C (\%) & $9.9 \pm 1.9$ & $9.3 \pm 1.5$ \\
\hline TC (mmol/L) & $4.8 \pm 1.2^{*}$ & $4.3 \pm 1.1$ \\
\hline LDL-C (mmol/L) & $2.7 \pm 1.1$ & $2.3 \pm 0.9$ \\
\hline HDL-C (mmol/L) & $1.3 \pm 0.4^{*}$ & $1.2 \pm 0.5$ \\
\hline TG (mmol/L) & $1.8 \pm 1.0$ & $1.8 \pm 1.5$ \\
\hline Apo $B^{2}(m g / L)$ & $1517.4 \pm 1188.2$ & $1556.4 \pm 1024.0$ \\
\hline Apo $A-I^{2}(m g / L)$ & $1799.7 \pm 330.4$ & $1687.7 \pm 446.6$ \\
\hline Apo A-II $(\mathrm{mg} / \mathrm{L})$ & $223.8 \pm 89.3$ & $230.8 \pm 99.6$ \\
\hline Apo C-II (mg/L) & $111.4 \pm 50.8$ & $116.7 \pm 61.2$ \\
\hline Apo C-III ${ }^{2}(\mathrm{mg} / \mathrm{L})$ & $331.1 \pm 202.8$ & $356.1 \pm 235.1$ \\
\hline Apo $E^{2}(\mathrm{mg} / \mathrm{L})$ & $92.9 \pm 60.7$ & $94.6 \pm 52.1$ \\
\hline Statins (\% of people prescribed) & 55.2 & 52.9 \\
\hline Exogenous insulin (\% of people prescribed) & 54.3 & 41.2 \\
\hline Hypoglycemic drugs (\% of people prescribed) & 89.6 & 93.3 \\
\hline
\end{tabular}

1 Values are mean $\pm S D$

*significantly different at $P<0.05$

${ }^{*} 2 n=49$ women and $n=19$ men.

Table 1: Anthropometrics, physical activity, blood pressure, plasma glucose, HbA1C, plasma lipids and apolipoproteins of Latinos with T2DM ${ }^{1}$

\begin{tabular}{|c|c|c|}
\hline Parameter & Women ( $n=93)$ & Men ( $n=33)$ \\
\hline Total energy (Kcal/d) & $1462.3 \pm 797.1$ & $1600.5 \pm 751.0$ \\
\hline Total fat $(g / d)$ & $45.4 \pm 31.0$ & $54.6 \pm 36.4$ \\
\hline Total fat (\% Energy) & $28.2 \pm 11.3$ & $29.1 \pm 12.7$ \\
\hline Saturated fat $(g / d)$ & $15.0 \pm 10$ & $17.9 \pm 15$ \\
\hline Monounsaturated fat $(g / d)$ & $16.2 \pm 11.2$ & $20.5 \pm 15.0$ \\
\hline Polyunsaturated fat $(g / d)$ & $10.4 \pm 10.4$ & $11.3 \pm 8.0$ \\
\hline Trans Fatty Acids (g/d) & $2.7 \pm 2.9$ & $2.5 \pm 2.8$ \\
\hline Omega-3 Fatty Acids (g/d) & $1.0 \pm 1.0$ & $1.4 \pm 1.4$ \\
\hline Dietary Cholesterol (mg/d) & $159.8 \pm 146.9$ & $261.0 \pm 256.5$ \\
\hline Total Carbohydrate (\% Energy) & $55.9 \pm 15.1$ & $52.4 \pm 15.4$ \\
\hline Total Carbohydrate (g/d) & $207.1 \pm 133.5$ & $199.2 \pm 89.9$ \\
\hline Refined grains (servings/d) & $2.0 \pm 2.4$ & $3.1 \pm 2.9^{*}$ \\
\hline Glycemic Index (glucose) & $61.6 \pm 8.5$ & $65.0 \pm 6.8^{*}$ \\
\hline Glycemic Load (glucose) & $119.1 \pm 86.7$ & $120.1 \pm 52.6$ \\
\hline Total fiber $(\mathrm{g} / \mathrm{d})$ & $14.1 \pm 13.3$ & $12.8 \pm 8.6$ \\
\hline Soluble fiber (g/d) & $3.7 \pm 3.3$ & $3.6 \pm 3.2$ \\
\hline Insoluble fiber (g/d) & $10.3 \pm 11.2$ & $8.5 \pm 5.7$ \\
\hline Total Protein $(g / d)$ & $59.1 \pm 39.0$ & $78.0 \pm 56.7$ \\
\hline Protein (\% Energy) & $16.6 \pm 7.1$ & $18.6 \pm 7.0$ \\
\hline Animal protein(g/d) & $37.2 \pm 29.8$ & $57.1 \pm 53.7$ \\
\hline
\end{tabular}

Table 2: Dietary intake of Latinos diagnosed with T2DM obtained from a single 24-Hs recall1. 
Citation: Calle MC, Vega-López S, Segura-Pérez S, Volek JS, Pérez-Escamilla R, et al. (2010) Low Plasma Hdl Cholesterol and Elevated C Reactive Protein further Increase Cardiovascular Disease Risk in Latinos with Type 2 Diabetes. J Diabetes Metab 1:109. doi:10.4172/21556156.1000109

Page 4 of 9

\begin{tabular}{|c|c|c|}
\hline Parameter & High-HDL ( $\mathrm{n}=32)$ & Low-HDL $(\mathrm{n}=36)$ \\
\hline Women (\%) & 45.8 & 54.2 \\
\hline Men (\%) & 58.8 & 41.2 \\
\hline Self-reported physically active (\%): $30 \mathrm{~min}$ of $P A \geq 5$ times $x$ week & 42.2 & 37.9 \\
\hline Alcohol (g/d) & $0.04 \pm 0.17$ & $0.03 \pm 0.09$ \\
\hline Total Cholesterol (mmol/L) & $4.89 \pm 1.2$ & $4.50 \pm 1.2$ \\
\hline Total triglycerides (mmol/L) & $1.46 \pm 0.9$ & $2.03 \pm 1.2^{*}$ \\
\hline VLDL diameter (nm) & $50.4 \pm 12.0$ & $51.4 \pm 8.1$ \\
\hline Total VLDL particles (mmol/L) & $69.4 \pm 49.4$ & $97.0 \pm 48.2^{*}$ \\
\hline Large VLDL (mmol/L) & $3.5 \pm 4.7$ & $7.4 \pm 8.3^{*}$ \\
\hline Medium VLDL (mmol/L) & $27.5 \pm 21.5$ & $47.1 \pm 30.8^{*}$ \\
\hline Small VLDL (mmol/L) & $38.4 \pm 30.7$ & $42.5 \pm 21.0$ \\
\hline IDL (mmol/L) & $73.2 \pm 74.9$ & $56.3 \pm 44.9$ \\
\hline Total LDL (mmol/L) & $2.62 \pm 1.0$ & $2.63 \pm 1.2$ \\
\hline LDL diameter (nm) & $21.2 \pm 1.0^{\star *}$ & $20.1 \pm 0.7$ \\
\hline Total LDL particles (mmol/L) & $1150.3 \pm 507.4^{*}$ & $1294.4 \pm 416.7$ \\
\hline Large LDL (mmol/L) & $404.8 \pm 197.6^{\star *}$ & $202.8 \pm 145.7$ \\
\hline Medium Small LDL (mmol/L) & $137.6 \pm 104.4^{\star *}$ & $204.8 \pm 90.9$ \\
\hline Very Small LDL (mmol/L) & $672.3 \pm 527.0^{\star *}$ & $1035.2 \pm 439.3$ \\
\hline Total HDL-C (mmol/L) & $1.46 \pm 0.5^{\star *}$ & $0.99 \pm 0.2$ \\
\hline HDL diameter $(\mathrm{nm})$ & $9.2 \pm 0.6^{* *}$ & $8.7 \pm 0.3$ \\
\hline Total HDL particles $(\mu \mathrm{mmol} / \mathrm{L})$ & $32.7 \pm 6.2^{*}$ & $28.7 \pm 5.1$ \\
\hline Large HDL ( $\mu \mathrm{mmol} / \mathrm{L})$ & $8.6 \pm 5.1^{* *}$ & $3.9 \pm 2.1$ \\
\hline Medium HDL ( $\mu \mathrm{mmol} / \mathrm{L})$ & $6.2 \pm 5.7$ & $4.7 \pm 4.6$ \\
\hline Small HDL ( $\mu \mathrm{mmol} / \mathrm{L})$ & $17.9 \pm 6.5$ & $20.2 \pm 6.6$ \\
\hline Statins (\% of people prescribed) & 51.6 & 57.8 \\
\hline
\end{tabular}

${ }^{1}$ Values are mean \pm SD Values

*Significantly different at $\mathrm{P}<0.05$

** Significantly different at $P<0.001$

Table 3: Total triglycerides, number and sizes of VLDL, IDL, LDL and HDL particles in Latinos diagnosed with T2DM according to plasma HDL-C levels: Low-HDL(<1.03 or $<1.3 \mathrm{mmol} / \mathrm{L}$ for men and women respectively) or High-HDL $(\geq 1.03 \text { or } \geq 1.3 \mathrm{mmol} / \mathrm{L} \text { for men and women respectively })^{1}$.

\begin{tabular}{|c|c|c|}
\hline Parameter & High-CRP' ( $\mathrm{n}=29)$ & Low-CRP $(\mathrm{n}=35)$ \\
\hline Women (\%) & 49.0 & 51.0 \\
\hline Men (\%) & 26.7 & 73.3 \\
\hline Self-reported physically active (\%): $30 \mathrm{~min}$ of $P A \geq 5$ times $x$ week & 26.1 & $73.9^{*}$ \\
\hline WC (cm) & $114.6 \pm 14.4^{* *}$ & $101.5 \pm 15.4$ \\
\hline Weight (kg) & $93.7 \pm 23.0$ & $78.3 \pm 23.0$ \\
\hline BMI $\left(\mathrm{kg} / \mathrm{m}^{2}\right)$ & $37.6 \pm 8.8^{\star \star}$ & $30.5 \pm 6.8$ \\
\hline Adiponectin (mg/L) & $3.9 \pm 2.9$ & $3.8 \pm 3.0$ \\
\hline Leptin (mg/L) & $3.7 \pm 2.0^{* *}$ & $2.7 \pm 2.6$ \\
\hline Insulin (pg/mL) & $134.2 \pm 81.1$ & $138.9 \pm 162.8$ \\
\hline CRP ( mg/L) & $8.3 \pm 6.4^{\star *}$ & $1.5 \pm 0.9$ \\
\hline TNF- $\alpha(\mu g / L)$ & $5.4 \pm 5.1$ & $4.9 \pm 2.9$ \\
\hline IL-6 (pg/mL) & $8.3 \pm 10.4$ & $6.1 \pm 5.2$ \\
\hline MCP-1 ( $\mu \mathrm{g} / \mathrm{L})$ & $195.4 \pm 79.1$ & $166.1 \pm 83.0$ \\
\hline sICAM ( $\mu \mathrm{g} / \mathrm{L})$ & $259.0 \pm 125.3^{*}$ & $201.6 \pm 125$ \\
\hline Statins (\% of people prescribed) & 48.3 & 57.1 \\
\hline Exogenous insulin (\% of people prescribed) & 48.3 & 55.9 \\
\hline Hypoglycemic drugs (\% of people prescribed) & 96.4 & 94.3 \\
\hline
\end{tabular}

${ }^{1}$ Values are mean \pm SD Values

*Significantly different at $\mathrm{P}<0.05$

** Significantly different at $P<0.001$

Table 4: Waist circumference (WC), weight, BMI, Apos, adiponectin, leptin, insulin, cytokines and chemokines in Latinos (men and women) diagnosed with T2DM according to CRP levels: High-CRP (> 3mg/L) or Low (<3mg/L). 
Citation: Calle MC, Vega-López S, Segura-Pérez S, Volek JS, Pérez-Escamilla R, et al. (2010) Low Plasma Hdl Cholesterol and Elevated C Reactive Protein further Increase Cardiovascular Disease Risk in Latinos with Type 2 Diabetes. J Diabetes Metab 1:109. doi:10.4172/21556156.1000109

Page 5 of 9

a two-tailed p-value $<0.05$. Pearson correlation coefficients were used to determine the association between CRP and leptin and WC. For Pearson correlations, CRP was analyzed as the natural logarithm.

\section{Results}

Anthropometrics, PA, plasma glucose and $\mathrm{HbA} 1 \mathrm{c}$ are presented in (Table 1) Age, blood pressure, BMI, WC, plasma glucose and HbA1c were not different between men and women. Body weight was lower in women $(82.6 \pm 21 \mathrm{~kg})$ compared to men $(91.6 \pm 23 \mathrm{~kg})(\mathrm{p}<0.05)$. However, all participants were obese based on their large BMI. A higher percentage of men (63.0\%) reported that they perform PA for at least 30 minutes on 5 or more days a week as compared to $32.5 \%$ of women $(\mathrm{p}<0.05)$. High plasma glucose and Hb1Ac levels indicated poor glycemic control.

Plasma lipids, apolipoproteins and medication use are shown in Table 1

Women had higher plasma total cholesterol than men $(4.8 \pm 1.2$ vs. $4.3 \pm 1.1 \mathrm{mmol} / \mathrm{L}$, respectively; $\mathrm{p}<0.05)$ and higher plasma HDL-C $(1.3 \pm 0.4$ vs. $1.2 \pm 0.5 \mathrm{mmol} / \mathrm{L}$, respectively; $\mathrm{p}<0.05)$. There were no differences between genders for plasma TG or for any of the apolipoproteins. Both women and men had high levels of Apo B $(1515.4 \pm 1188.2$ and $1556.4 \pm 1024.0 \mathrm{mg} / \mathrm{L}$ respectively) and high levels of Apo CIII (331.1 \pm 202.8 and $356.1 \pm 235.1 \mathrm{mg} / \mathrm{L}$, respectively). There were no significant differences between women and men regarding statins prescription, hypoglycemic drugs or exogenous insulin use.

Diet analysis: Total energy, macronutrients, total fiber (soluble and insoluble), total sugar, glycemic index and glycemic load, total cholesterol, saturated fat, TRANS fat, polyunsaturated fatty acids (PUFA) and monounsaturated fatty acids (MUFA) were analyzed (Table 2). There were no differences between men and women in macronutrient intake. Regarding food groups men consumed more refined grains servings per day $(3.1 \pm 2.9)$ than women $(2.0 \pm 2.4)$ $(\mathrm{p}<0.05)$. Consistently the glycemic index (glucose) was significantly higher in men $(65.0 \pm 6.8)$ than women $(61.6 \pm 8.5)(\mathrm{p}<0.05)$. The glycemic load was high in both genders $(119.1 \pm 86.7$ and $120.1 \pm$ 52.6 respectively). Additionally both women $(14.1 \pm 13.3 \mathrm{~g} / \mathrm{d})$ and men $(12.8 \pm 8.6 \mathrm{~g} / \mathrm{d})$ had low intakes of dietary fiber.

The data analyses for lipoprotein particle size and number, apolipoproteins and cytokines were performed in a smaller subsample of the baseline DIALBEST trial. The rationale for the selection criteria was the order of enrollment, thus the first 68 participants enrolled in DIALBEST were included. There were no differences in the anthropometric or plasma lipid characteristics of these 68 participants with the rest of the subsample in our study (data not shown).

Participants $(\mathrm{n}=68)$ were divided according to their plasma HDL-C levels into Low-HDL $(<1.03$ or $<1.3 \mathrm{mmol} / \mathrm{L}$ for men and women, respectively) and High-HDL $(\geq 1.03$ or $\geq 1.3 \mathrm{mmol} / \mathrm{L}$ for men and women respectively) and the number and size of lipoproteins were analyzed (Table 3). The Low-HDL group had higher TG, more total VLDL particles as well as large and medium VLDL than the High$H D L$ group $(\mathrm{p}<0.05)$. Consistently, the $L o w-H D L$ group had smaller LDL diameter $(\mathrm{p}<0.001)$, higher concentrations of small LDL $(\mathrm{P}<$ $0.0001)$ and lower number of the large LDL particles $(\mathrm{p}<0.001)$ than the High-HDL group. As expected, the Low-HDL group had a smaller HDL diameter than the High HDL group $(\mathrm{p}<0.001)$ as well as a lower number of the large and more protective HDL particles $(\mathrm{p}<0.001)$.
Finally, by definition the $L o w-H D L$ group had a lower number of total HDL particles $(\mathrm{p}<0.05)$ and total HDL-C $(\mathrm{p}<0.00001)$ than the High$H D L$ group. Interestingly, plasma LDL-C concentrations were not different between HDL groups emphasizing that low HDL-C is highly associated with atherogenic lipoproteins independent of plasma LDL-C. There were not differences on the percentage of people prescribed with statins between HDL groups.

Plasma cytokines: subjects $(n=68)$ were divided by their chronic inflammatory status according to CRP levels: $\operatorname{High}-C R P(\geq 3 \mathrm{mg} / \mathrm{L})$ or Low-CRP $(<3 \mathrm{mg} / \mathrm{L})$. Latinos from the High-CRP group had larger WC $(\mathrm{p}<0.001)$ and higher BMI $(\mathrm{p}<0.001)$ than those in the Low-CRP group (Table 4). In addition, a positive correlation was found between WC and CRP levels ( $r=0.49, p<0.05$; Figure 1). These data support the association of adipose tissue with chronic low grade inflammation. Interestingly, only approximately $1 / 4$ of the men compared to $1 / 2$ of the women were grouped in the High-CRP group. The percentage of people who reported to be more physically active was lower in the High-CRP group (26.1\%) compared to the Low-CRP group (73.9\%, $\mathrm{p}<0.05)$. Latinos in the High-CRP group had higher leptin levels

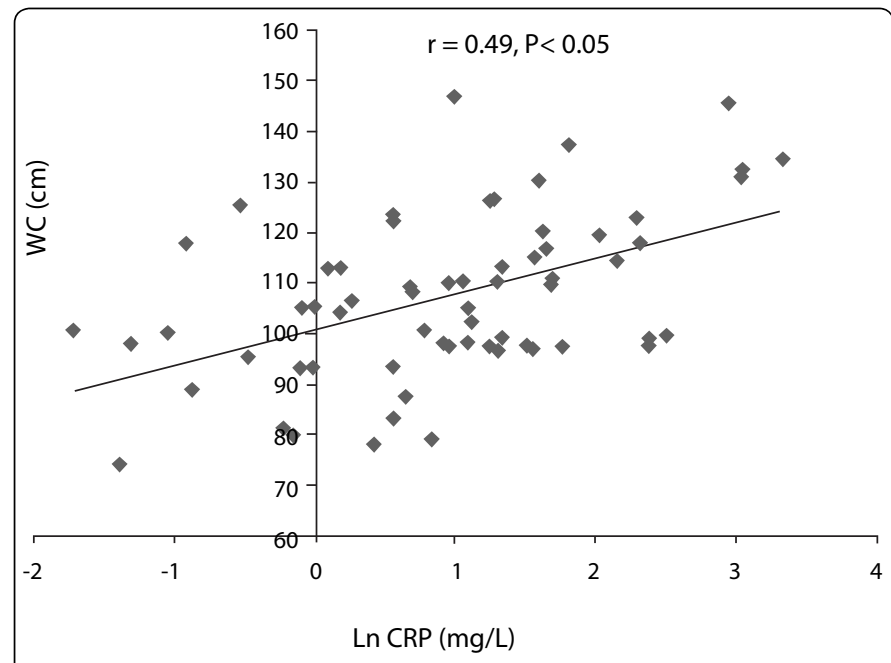

Figure 1: Waist circumference positively correlates with plasma CRP in Latinos diagnosed with type 2 diabetes $(r=0.49, p<0.05)$.

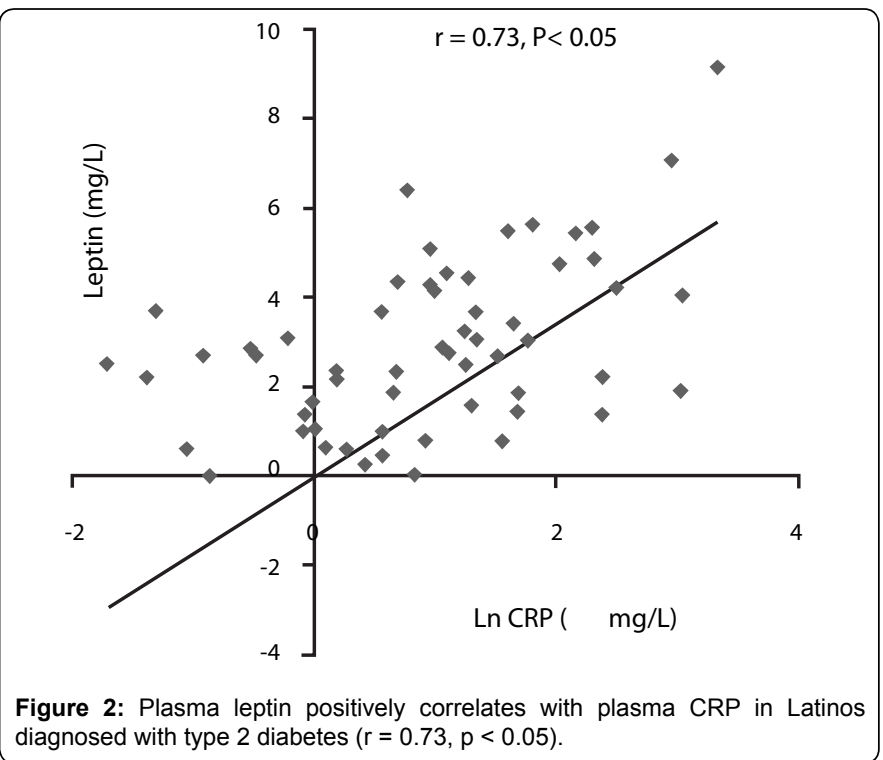


Citation: Calle MC, Vega-López S, Segura-Pérez S, Volek JS, Pérez-Escamilla R, et al. (2010) Low Plasma Hdl Cholesterol and Elevated C Reactive Protein further Increase Cardiovascular Disease Risk in Latinos with Type 2 Diabetes. J Diabetes Metab 1:109. doi:10.4172/21556156.1000109

Page 6 of 9

$(3.7 \pm 2.0 \mathrm{mg} / \mathrm{L})$ than those in the Low-CRP group $(2.7 \pm 2.6, \mathrm{p}<0.001)$. In addition, there was a strong positive correlation between plasma leptin and CRP levels $(r=0.73, p<0.05$; Figure 2). sICAM levels were significantly higher in the High-CRP group $(259.0 \pm 125.3 \mu \mathrm{g} / \mathrm{L})$ compared to the Low-CRP group $(201.6 \pm 125 \mu \mathrm{g} / \mathrm{L}, \mathrm{p}<0.05)$. Lastly, by definition CRP levels were significantly higher in the High-CRP group $(\mathrm{p}<0.001)$. There were no differences on statin prescription, hypoglycemic drugs or exogenous insulin use between the High and Low CRP groups.

\section{Discussion}

In this study we have demonstrated that in Latinos diagnosed with type 2 diabetes, high HDL-C as well as lower concentrations of CRP appear to be somewhat protective in this population already at risk for CVD. Low concentrations of HDL-C as well as high concentrations of CRP were accompanied by other markers of increased CVD risk in this already challenged population. However these two conditions appear to be independent of each other because low HDL-C and high CRP or vice versa were not significantly correlated in this population. Participants with low HDL-C had higher concentrations of atherogenic lipoproteins and smaller HDL particles associated with less efficient reverse cholesterol transport. Meanwhile those with high CRP had more fat accumulated in the trunk area and higher concentrations of leptin and sICAM, a marker of atherosclerosis. These data suggest that both HDL-C at lower concentrations than the recommendations as well as high CRP are key markers of more advanced complications in subjects with type-2 diabetes.

Gender comparisons for traditional CVD risks factors: anthropometrics, plasma lipids, apolipoproteins and dietary intake analysis

The cutoff point value by the NCEP ATP III to determine central adiposity is $88 \mathrm{~cm}$ and $102 \mathrm{~cm}$ for women and men respectively [29]. Mean waist circumference (WC) for women was $106.3 \pm 16.0 \mathrm{~cm}$ and $109.7 \pm 17.4 \mathrm{~cm}$ for men, respectively. These data along with the BMI indicate that this Latino population is obese and that they have excessive central adiposity. The WC was not different between men and women. However, women were further off the $88 \mathrm{~cm}$ compared to the $102 \mathrm{~cm}$ for men suggesting a higher accumulation of trunk fat. Obese people tend to overestimate their PA when self reported data are used [30]. In agreement with their obesity rate, this Latino group might be more sedentary than what they have reported. The data indicate that men are more physically active that women, this is consistent with previous PA patterns reported in Latinos [31]. Approximately $62.3 \%$ of the participants were prescribed with medications to control blood pressure, such as beta blockers or angiotensin converting enzyme (ACE) inhibitors at enrollment. This might explain why the blood pressure fell within the normal range. The levels of plasma glucose from our study were slightly higher than those found in Hispanics with T2D [ADOPT study $(8.5 \pm 1.7 \mathrm{mmol} / \mathrm{L})$ [32]; NHANES III $(5.03 \pm 0.04 \mathrm{mmol} / \mathrm{L})$ [33]. Along with the high HbA1c levels reported in our subjects, these data indicate poor T2D control in this Latino group. Women have higher HDL-C than men by definition and our data confirmed that fact. However, women with an HDL-C of $1.3 \pm 0.4 \mathrm{mg} / \mathrm{dL}$ were barely above the NCEP ATP III cutoff point for normal HDL-C $(\geq 1.3 \mathrm{mg} / \mathrm{dL})$. Meanwhile men with $1.2 \pm 0.5 \mathrm{mg} / \mathrm{dL}$ were far above the normal cutoff point $(\geq 1.03 \mathrm{mg} /$ $\mathrm{dL})$. This fact, in combination with the slightly higher plasma total cholesterol indicates that women might be at higher risk for CVD than men in these group of Latino participants.
We also analyzed dietary intake of study participants. The low number of calories suggests underreport from most of the participants, which is consistent with reported dietary assessment from obese people [34]. The macronutrient composition and cholesterol intake of this Latino population was in agreement with The United States Department of Agriculture (USDA). However, this population had low fiber intake, with moderate glycemic index and high glycemic load. Furthermore, trans fatty acid intake in this T2D group was higher than the $1 \%$ recommended by USDA. Neither the amount of dietary carbohydrate nor the glycemic load were different between men and women.

Apolipoproteins are exchangeable between lipoproteins particles, except for apo B which remains in VLD-LDL particles [35]. There were no differences in the apolipoproteins between genders. The Apo AI, Apo B, Apo CII, Apo CIII and Apo E values reported in this study are consistent with those found in obese subjects in our laboratory [36]. The high levels of Apo B in this T2D group suggest the presence of small LDL particles consistent with a more atherogenic lipid profile or Pattern B as was confirmed by the high number of small LDL particles. Thus although LDL-C concentrations were within the normal range, there was a high number of small LDL particles. Apo CIII can impair VLDL lipolysis, through the inhibition of Apo CII and as a result the inhibition of lipoprotein lipase [37]. The high Apo CIII levels in these Latinos are related to a deregulation of TG metabolism.

In addition to the more pronounced dyslipidemias, women were less physically active and had higher central adiposity than men suggesting that in this population of diabetic individuals, women might be at a higher CVD risk.

\section{Plasma lipids in latinos diagnosed with T2D}

The plasma lipids profile in T2D is characterized by low plasma HDL-C and very high triglycerides [38]. Plasma triglycerides reported in our study were slightly higher than the desirable $1.7 \mathrm{mmol} / \mathrm{L}$ according to ATPIII guidelines [29]. However, the reported plasma HDL-C was within the normal range. Other studies in Latinos diagnosed with T2D reported lower HDL-C concentrations than those observed in the current study $(1.1 \pm 0.1$ and $1.2 \pm 0.04 \mathrm{mmol} / \mathrm{L}$, respectively $)$ $[32,39]$. One of the possible reasons for these differences could be that at least half of the participants in our study were prescribed with statins. Besides lowering LDL-C through the inhibition of hydroxymethylglutaryl coenzyme A reductase (rate limiting enzyme for cholesterol synthesis), statins may also affect plasma triglycerides and HDL [40]. Still the increases in HDL-C by statins are modest (4-12\%) [41]. Even though the mean plasma lipids were apparently normal, when we divided the subjects into Low or High HDL-C levels, approximately half of them presented the type of dyslipidemia associated with T2D. Overall, in this subsample of Latino that seems to have plasma lipids within the normal range, there are individuals that might require a different therapeutic approach.

\section{Low HDL-C level is associated with a more atherogenic lipoprotein profile}

Larger HDL has longer plasma half life and is more effective in reverse cholesterol transport [2]. The Low-HDL group had smaller HDL diameter and fewer number of the large HDL molecules. These data is consistent with HDL characteristics reported in diabetic subjects [21]. Thus, Latinos in the Low-HDL group might have impaired reverse cholesterol transport which places them at a higher CVD risk. Large VLDL carries more cholesterol esters than small VLDL. The secretion rate of larger VLDL is dependent on TG availability. As 
Citation: Calle MC, Vega-López S, Segura-Pérez S, Volek JS, Pérez-Escamilla R, et al. (2010) Low Plasma Hdl Cholesterol and Elevated C Reactive Protein further Increase Cardiovascular Disease Risk in Latinos with Type 2 Diabetes. J Diabetes Metab 1:109. doi:10.4172/21556156.1000109

Page 7 of 9

expected by the higher TG levels, the Low-HDL group had more of the large and medium VLDL than the High-HDL group. After lipoprotein lipase action, large VLDL particles become small LDL particles [42]. Indeed, the Low-HDL group had smaller LDL particles. Small dense LDL particles have been associated with higher risk of developing CVD [43]. Small LDL is considered more atherogenic because its decreased affinity to bind to the LDL receptor and its susceptibility to oxidative modification [27]. This modification increases the propensity for small LDL transport into the sub endothelial space and binding to the arterial proteoglycans [9]. Therefore, it is necessary to take into account the abnormalities in lipoprotein particles size in this subset of Latinos with T2D when evaluating CVD risk and potential treatments.

Surprisingly, there were no differences on the percentage of people prescribed with statins within High or Low-HDL groups. This could be explained because the main target of statins is lowering LDL-C [29] although a modest effect has been shown in HDL-C. In this specific group of Latinos with low HDL-C and high TG, treating only LDL-C levels might disregard significant excess risk for CVD. On the other hand, fibrates positively impact triglyceride metabolism and as a result HDL-C [44]. Fibrates are a peroxisomal proliferator activator receptors alpha (PPAR- $\alpha$ ) agonist that have good results at improving the dyslipidemia characteristic of T2D and the atherogenic lipoproteins particle size [45]. Interestingly, only $5 \%$ of the participants in this study were prescribed with fibrates, (data not shown) as compared to $55 \%$ prescribed with statins. Thus, fibrates therapy might be helpful for Latinos with T2D with this atherogenic lipoprotein profile. The efficacy of using statins and fibrates combined is still not clear and it may depend of the different types of drugs or combinations used. Some studies reported that combination therapy has been effective at improving LDL size in people with T2D [46]. However, some recent randomized trials showed no further improvements in CVD risk by using the combined therapy over monotherapy $[47,48]$. Furthermore, combined therapy may increase the risk of myopathy.

Overall these data suggest that low HDL-C in this diabetic population is associated with higher concentrations of large and medium VLDL, smaller LDL and smaller less functional HDL. This is consistent with an atherogenic lipoprotein profile or pattern B. A change in life style, including weight management and better control of their disease may be beneficial to these Latino subjects with Low HDL-C to ameliorate their CVD risk.

\section{High inflammatory status increases CVD risk in latinos diagnosed with T2D}

Low grade inflammation is a common feature in T2D subjects [22]. CRP is a well establish marker for CVD risk and inflammation [49]. CRP facilitates LDL uptake by macrophages and contributes to endothelial dysfunction [50]. CRP is produced mostly by the liver upon stimulation of IL- 6 and TNF- $\alpha$ [14]. Indeed, although not significant, there were higher levels of these cytokines in the HighCRP group. This study may have lacked statistical power to detect these differences. CRP increases the endothelial cells production of sICAM-1 [15] and MCP-1[16]. These molecules are involved in the development of atherosclerosis [17]. sICAM and MCP1 levels in the High-CRP group were consistent with those found in obese subjects in our Laboratory [51]. In our study Latinos in the High-CRP group had higher sICAM levels than the Low CRP. sICAM mediates the adhesion of mononuclear cells to the endothelium to enter in the sub endothelial space [52]. Overall, those with High CRP levels are at higher CVD risk as shown by increased levels on markers of atherosclerosis, such as SICAM.
Central obesity is one of the features associated with CRP, consistently in our study CRP levels positively correlates with WC. As expected those in the High-CRP group had higher WC and were more obese. Physical activity has been associated with reduction in CRP in patients with type 2 diabetes independent of weight loss [53]. Latinos in the $L O W$-CRP group reported to be more physically active than those in the High-CRP group. Moreover being more physically active might prevent further body fat accumulation, which is associated with higher inflammatory status. Only $1 / 4$ of the men as compared to $1 / 2$ of the women were clustered in the High-CRP group. Coincidently, women had higher WC than men. Overall, central adiposity was closely linked to high CRP levels in these subjects.

Obese people tend to have high plasma leptin levels [54]. Indeed, Latinos in the high CRP group, which were also more obese, had higher leptin levels. Additionally in our study, plasma leptin positively correlated with plasma CRP levels. Our data suggest that this population could be leptin resistant. In vitro and in vivo experiments support the hypothesis that CRP can induce leptin resistance by binding to leptin and impairing its signaling [55]. Adiponectin was within the low range probably preventing the protective action of this adypokine. Adiponectin is associated with insulin sensitivity and with reduced risk for atherosclerosis [18]. Low adiponectin may impact plasma HDL-C, since adiponectin has been inversely correlated with HDL-apoA1 catabolism [56]. Plasma insulin was within the normal ranges and there were no differences in plasma insulin between groups. This could have been due to the high standard deviation because they were receiving exogenous insulin. Our assay did not differentiate between endogenous and exogenous insulin. Even though not significant, a higher number of Latinos from the Low-CRP group have been prescribed with statins and were using exogenous insulin. The pleiotropic effects of statins [57] and the anti-inflammatory effect of insulin [58] might have contributed to the differences in inflammatory status. In summary, the low adiponectin levels for all the Latinos might prevent the otherwise CVD protection from this adypokine. The high leptin levels in the High-CRP group suggest a derangement in energy balance metabolism as confirmed by the higher WC and higher number of obese individuals.

\section{Conclusions}

Results from this study indicate that individuals with T2D and healthy HDL concentrations appear to be more protected against CVD risk as indicated by the lower number of atherogenic particles including large and medium VLDL as well as small LDL. In addition, LDL size in the high-HDL group is more representative of pattern $\mathrm{A}$, associated with decreased risk for CVD. Similarly, individuals having lower concentrations of CRP have a BMI that can be considered borderline overweight $\left(30.5 \mathrm{~kg} / \mathrm{m}^{2}\right)$ compared to the high-CRP group $\left(37.6 \mathrm{~kg} / \mathrm{m}^{2}\right)$, which is in the upper range of obese. Further, leptin values in the low CRP group are not associated with leptin resistance. Although both low HDL and high CRP have been identified as factors associated with increased risk for CVD, data from this study clearly demonstrates that T2D subjects with normal HDL and CRP values might be more protected against atherosclerosis and heart disease.

\section{Acknowledgment}

This work was partially funded by the Connecticut NIH EXPORT Center for Eliminating Health Disparities among Latinos, grant \# NIH-NCMHD P20MD001765, awarded to RPE. Finally, special thanks to all who participated in the study and to community health care workers at the Hispanic Health Council.

\section{References}

1. Lloyd-Jones D, Adams R, Carnethon M, De Simone G, Ferguson TB, et al. 
Citation: Calle MC, Vega-López S, Segura-Pérez S, Volek JS, Pérez-Escamilla R, et al. (2010) Low Plasma Hdl Cholesterol and Elevated C Reactive Protein further Increase Cardiovascular Disease Risk in Latinos with Type 2 Diabetes. J Diabetes Metab 1:109. doi:10.4172/21556156.1000109

Page 8 of 9

(2009) Heart disease and stroke statistics--2009 update: a report from the American Heart Association Statistics Committee and Stroke Statistics Subcommittee. Circulation 119: e21-181.

2. Cardenas GA, Lavie CJ, Cardenas V, Milani RV, McCullough PA (2008) The importance of recognizing and treating low levels of high-density lipoprotein cholesterol: A new era in atherosclerosis management. Rev Cardiovasc Med 9: 239-258.

3. Nofer JR, Walter M, Kehrel B, Wierwille S, Tepel M, et al. (1998) HDL3mediated inhibition of thrombin-induced platelet aggregation and fibrinogen binding occurs via decreased production of phosphoinositide- derived second messengers 1,2-diacylglycerol and inositol 1,4,5-tris- phosphate. Arterioscler Thromb Vasc Biol 18: 861-869.

4. Aviram M, Rosenblat M, Bisgaier CL, Newton RS, Primo-Parmo SL, et al. (1998) Paraoxonase inhibits high-density lipoprotein oxidation and preserves its functions: A possible peroxidative role for paraoxonase. J Clin Invest 101: $1581-1590$.

5. Yu Bl, Wang Sh, Peng Dq, Zhao Sp (2010) HDL and immunomodulation: an emerging role of HDL against atherosclerosis. Immunol Cell Biol 88: 285-290.

6. Nofer JR, Van Der Giet M, Tölle M, Wolinska I, Von Wnuck Lipinski K, et al. (2004) HDL induces NO-dependent vasorelaxation via the lysophospholipid receptor S1P3. J Clin Invest 113: 569-581.

7. Tenenbaum A, Fisman EZ, Motro M, Adler Y (2006) Atherogenic dyslipidemia in metabolic syndrome and type 2 diabetes: Therapeutic options beyond statins. Cardiovasc Diabetolo5: 20

8. Krauss RM, Burke DJ (1982) Identification of multiple subclasses of plasma low density lipoproteins in normal humans. J Lipid Res 23: 97-104.

9. Berneis KK, Krauss RM (2002) Metabolic origins and clinical significance of LDL heterogeneity. J Lipid Res 43: 1363-1379.

10. Björnheden T, Babyi A, Bondjers G, Wiklund O (1996) Accumulation of lipoprotein fractions and subfractions in the arterial wall, determined in an in vitro perfusion system. Atherosclerosis 123: 43-56.

11. Blasi C (2008) The autoimmune origin of atherosclerosis. Atherosclerosis 201 17-32.

12. Harangi M, Szodoray P, Paragh G (2009) Atherosclerosis: A complex interplay of inflammatory processes. Future Medicine 4: 167-187.

13. Broncel M, Kozirol GM, Chojnowska-Jezierska J (2008) Lipid lowering agents inflammation and atherosclerosis. Antiinflamm Antiallergy Agents Med Chem 7: 206-216.

14. Devaraj S, Singh U, Jialal I (2009) The evolving role of C-reactive protein in atherothrombosis. Clin Chem 55: 229-238.

15. Pasceri V, Willerson JT, Yeh ET (2000) Direct proinflammatory effect of C-reactive protein on human endothelial cells. Circulation 102: 2165-2168.

16. Pasceri V, Chang J, Willerson JT, Yeh ET (2001) Modulation of C-reactive protein-mediated monocyte chemoattractant protein-1 induction in human endothelial cells by anti-atherosclerosis drugs. Circulation 103: 2531-2534.

17. Alexandraki K, Piperi C, Kalofoutis C, Singh J, Alaveras A, et al. (2006) Inflammatory process in type 2 diabetes: The role of cytokines. Ann N Y Acad Sci1084: 89-117.

18. Gualillo O, Gonzal lez-Juanatey JR, Lago F (2007) The Emerging Role of Adipokines as Mediators of Cardiovascular Function: Physiologic and Clinical Perspectives. Trends Cardiovascu Med 17: 275-283.

19. Ong KL, Cheung BMY, Wong LYF, Wat NMS, Tan KCB, et al. (2008) Prevalence Treatment, and Control of Diagnosed Diabetes in the U.S. National Health and Nutrition Examination Survey 1999-2004. Ann Epidemiol 18: 222-229.

20. Fox CS, Coady S, Sorlie PD, Levy D, Meigs JB, et al. (2004) Trends in cardiovascular complications of diabetes. JAMA 292: 2495-2499.

21. Vergès $B$ (2009) Lipid modification in type 2 diabetes: The role of LDL and HDL. Fundam Clin Pharmacol 23: 681-685.

22. Pickup JC (2004) Inflammation and activated innate immunity in the pathogenesis of type 2 diabetes. Diabetes Care 27: 813-823.

23. Pérez-Escamilla R, Hromi-Fiedler A, Vega-López S, Bermudez-Millan A Segura-Pérez S (2008) Impact of Peer Nutrition Education on Dietary Behaviors and Health Outcomes among Latinos: A Systematic Literature Review. J Nutr Educ Behav 40: 208-225.
24. Little RR (2005) Point-of-care testing for glycated hemoglobin (GHB). Diabetes Technol Ther 7: 913-915

25. Allain CC, Poon LS, Chan CS, Richmond W, Fu PC (1974) Enzymatic determination of total serum cholesterol. Clin Chem 20: 470-475.

26. Friedewald WT, Levy RI, Fredrickson DS (1972) Estimation of the concentration of low-density lipoprotein cholesterol in plasma, without use of the preparative ultracentrifuge. Clin Chem 18: 499-502.

27. Wood RJ, Volek JS, Liu Y, Shachter NS, Contois JH, et al. (2006) Carbohydrate restriction alters lipoprotein metabolism by modifying VLDL, LDL, and HDL subfraction distribution and size in overweight men. J Nutr 136: 384-389.

28. Liu S, Tinker L, Song Y, Rifai N, Bonds DE, et al. (2007) A prospective study of inflammatory cytokines and diabetes mellitus in a multiethnic cohort of postmenopausal women. Arch Intern Med 167: 1676-1685

29. Executive summary of the third report of the National Cholesterol Education Program (NCEP) expert panel on detection, evaluation, and treatment of high blood cholesterol in adults (adult treatment panel III). (2001) JAMA 285: 24862497.

30. Prince SA, Adamo KB, Hamel ME, Hardt J, Connor Gorber S, et al. (2008) A comparison of direct versus self-report measures for assessing physical activity in adults: A systematic review. Int J Behavl Nutr Phys Act 5: 56

31. Marquez DX, McAuley E (2006) Gender and acculturation influences on physical activity in Latino adults. Ann BehavMed 31: 138-144.

32. Viberti G, Lachin J, Holman R, Zinman B, Haffner S, et al. (2006) A Diabetes Outcome Progression Trial (ADOPT): Baseline characteristics of Type 2 diabetic patients in North America and Europe. Diabet Med 23: 1289-1294.

33. Chiu KC, Martinez DS, Chu A (2005) Comparison of the relationship of age and beta cell function in three ethnic groups. Clin Endocrinol 62: 296-302.

34. Lichtman SW, Pisarska K, Berman ER, Pestone M, Dowling H, et al. (1992) Discrepancy between self-reported and actual caloric intake and exercise in obese subjects. N Engl J Medi 327: 1893-1898.

35. Borggreve SE, De Vries R, Dullaart RPF (2003) Alterations in high-density lipoprotein metabolism and reverse cholesterol transport in insulin resistance and type 2 diabetes mellitus: Role of lipolytic enzymes, lecithin:cholesterol acyltransferase and lipid transfer proteins. Eur J Clin Inves 33: 1051-1069.

36. Mutungi G, Waters D, Ratliff J, Puglisi M, Clark RM, et al. (2009) Eggs distinctly modulate plasma carotenoid and lipoprotein subclasses in adult men following a carbohydrate-restricted diet. J Nutr Biochem 21: 261-267.

37. Yuan G, Chen X, Ma Q, Qiao J, Li R, et al. (2007) C-reactive protein inhibits adiponectin gene expression and secretion in 3T3-L1 adipocytes. J Endocrinol 194: $275-281$.

38. Verges B, Brun JM, Vaillant G, Quantin C, Brunet-Lecomte P, et al. (1992) Influence of obesity and hypertriglyceridaemia on the low HDL2-cholestero level and on its relationship with prevalence of atherosclerosis in Type 2 diabetes. Diabete Metab 18: 289-297.

39. Tull ES, Thurland A (2004) Dyslipidemia and Insulin Resistance in Relation to Genetic Admixture among Hispanics and Non-Hispanic Blacks of Caribbean Origin. J Natl Med Assoc 96: 332-340.

40. Dergunov AD, Visvikis-Siest S, Siest G (2008) Statins as effectors of key activities involved in apoE-dependent VLDL metabolism: Review and hypothesis. Vascul Pharmacol 48: 70-75.

41. Davidson MH (2002) Rosuvastatin: a highly efficacious statin for the treatment of dyslipidaemia. Expert Opin Investig Drugs 11: 125-141.

42. Rizzo M, Berneis K, Corrado E, Novo S (2006) The significance of low-densitylipoproteins size in vascular diseases. Int Angiol 25: 4-9.

43. Shrestha S, Freake HC, McGrane MM, Volek JS, Fernandez ML (2007) A combination of psyllium and plant sterols alters lipoprotein metabolism in hypercholesterolemic subjects by modifying the intravascular processing of lipoproteins and increasing LDL uptake. J Nutr 137: 1165-1170.

44. Van Raalte DH, Li M, Pritchard PH, Wasan KM (2004) Peroxisome proliferator activated receptor (PPAR)-a: A pharmacological target with a promising future. Pharm Res 21: 1531-1538.

45. Rizzo M, Rini GB, Berneis K (2007) The clinical relevance of LDL size and subclasses modulation in patients with type-2 diabetes. Exp Clin Endocrino Diabetes 115: 477-482. 
Citation: Calle MC, Vega-López S, Segura-Pérez S, Volek JS, Pérez-Escamilla R, et al. (2010) Low Plasma Hdl Cholesterol and Elevated C Reactive Protein further Increase Cardiovascular Disease Risk in Latinos with Type 2 Diabetes. J Diabetes Metab 1:109. doi:10.4172/21556156.1000109

Page 9 of 9

46. Grundy SM, Vega GL, Yuan Z, Battisti WP, Brady WE, et al. (2005) Effectiveness and tolerability of simvastatin plus fenofibrate for combined hyperlipidemia (the SAFARI trial). Am J Cardiol 95: 462-468

47. Stojakovic T, De Campo A, Scharnagl H, Sourij H, Schmölzer I, et al. (2010) Differential effects of fluvastatin alone or in combination with ezetimibe on lipoprotein subfractions in patients at high risk of coronary events. Eur $\mathrm{J}$ Clin Invest 40: 187-194.

48. Ginsberg HN, Elam MB, Lovato LC, Crouse lii JR, Leiter LA, et al. (2010) Effects of combination lipid therapy in type 2 diabetes mellitus. $\mathrm{N}$ Engl $\mathrm{J}$ Med 362: 1563-1574.

49. Pannacciulli N, Cantatore FP, Minenna A, Bellacicco M, Giorgino R, et al. (2001) C-reactive protein is independently associated with total body fat, central fat, and insulin resistance in adult women. Int J Obes Relat Metab Disord 25: 14161420.

50. Calabrò P, Golia E, Yeh ET (2009) CRP and the risk of atherosclerotic events. Semin Immunopathol 31: 79-94.

51. Al Sarraj T SH, Calle MC, Volek JS, Fernandez ML. (2009) Carbohydrate restriction, as a first-line dietary intervention, effectively reduces biomarkers of metabolic syndrome in Emirati adults. J Nutr 139: 1667-1676.
52. Lawson C, Wolf S (2009) ICAM-1 signaling in endothelial cells. Pharmacol Rep 61: 22-32.

53. Balducci S, Zanuso S, Nicolucci A, Fernando F, Cavallo S, et al. (2009) Antiinflammatory effect of exercise training in subjects with type 2 diabetes and the metabolic syndrome is dependent on exercise modalities and independent of weight loss. Nutr Metab Cardiovasc Dis 20: 608-617.

54. Martin SS, Qasim A, Reilly MP (2008) Leptin Resistance. A Possible Interface of Inflammation and Metabolism in Obesity-Related Cardiovascular Disease. J Am Coll Cardiol 52: 1201-1210.

55. Chen K, Li F, Li J, Cai H, Strom S, et al. (2006) Induction of leptin resistance through direct interaction of C-reactive protein with leptin. Nat Med 12: 425-432.

56. Vergès B, Petit JM, Duvillard L, Dautin G, Florentin E, et al. (2006) Adiponectin is an important determinant of ApoA-I catabolism. Arterioscler Thromb Vasc Biol 26: 1364-1369.

57. Martin JH, Mangiafico S, Kelly DJ (2009) Role of statins in diabetes complications. Curr Diabetes Rev 5: 165-170.

58. Dandona P, Chaudhuri A, Mohanty P, Ghanim H (2007) Anti-inflammatory effects of insulin. Curr Opin Clin Nutr Metab Care 10: 511-517. 